\title{
Using Analytic Morphomics to Predict Long-Term Risk in Kidney Donors
}

Jake Claflin ${ }^{1}$ and Mitchell B. Alameddine ${ }^{1}$; David C. Cron ${ }^{1}$; Michael N. Terjimanian ${ }^{1}$; Jeffrey F. Friedman ${ }^{1}$; Jennifer D. Klei ${ }^{1}$; Calista M. Harbaugh ${ }^{1}$; Adnan Hussain ${ }^{1}$; Seth A. Waits ${ }^{1}$; Stewart C. Wang ${ }^{1}$; Michael J. Englesbe ${ }^{1}$

\section{ABSTRACT}

\section{Introduction:}

Complications of chronic illnesses can take decades to develop, calling into question whether truly low-risk kidney donors can be reliably identified. Previous studies have demonstrated the potential benefit of analytic morphomics as a long-term risk assessment tool. We hypothesize that in kidney donors without known comorbidities, predonation morphomic measures will be associated with the development of chronic disease.

\section{Methods:}

All University of Michigan kidney donors from 2000 to 2010 with no known cardiovascular risk factors $(1,038)$ received a telephone interview. The survey specifically targeted the development of cardiovascular risk factors. Morphomic measures were determined using predonation computed tomography.

\section{Results:}

Survey results were obtained from 598 living kidney donors (response rate 57.6\%). The mean follow-up time from donation was 9.2 years. A total of $17.0 \%$ of patients reported new diagnosis of at least one cardiovascular risk factor including diabetes (2.5\%), hypertension (14.0\%), kidney disease (1.0\%), heart attack (0.3\%), and stroke (1.3\%). Morphometric age, a composite of several morphomic measures, correlated significantly with onset of cardiovascular risk factors ( $P=0.045)$ while chronological age did not $(P$ $=0.09$ ).

\section{Conclusions:}

Analytic morphomics does not clearly add to current methods of risk stratification in kidney donors, but it does show promise as a novel risk assessment tool. 


\section{Introduction}

In response to growing demand for life-saving kidney transplants, transplant centers have expanded living kidney donation and relaxed criteria for living donors, allowing older individuals and those with a modest burden of comorbidities to donate. ${ }^{1}$ Current studies suggest that these potentially higherrisk donors do not exhibit higher rates of long-term complications; however, long-term complications do develop in some living kidney donors. ${ }^{2-5} \mathrm{Com}$ plications of chronic illnesses such as hypertension or diabetes can take decades to develop, calling into question whether truly low-risk kidney donors can be reliably identified. Follow-up with kidney donors is minimal, and there are currently no methods by which to identify individuals who will develop long-term complications., 4,6,7

All potential kidney donors undergo a comprehensive screening that aims to allow only healthy individuals to undergo donor nephrectomy. These screening methods identify patients with clinically known risk factors such as hypertension or kidney disease. However, among individuals with no clinically known cardiovascular risk, a subset of patients with clinically silent risk factors may exist.4,7,8 Previous studies have validated analytic morphomics, which utilizes preoperative computed tomography (CT) scans as a novel risk stratification tool in various surgical populations. ${ }^{9-13}$ Analytic morphomics may be an especially useful risk stratification tool in living kidney donors because each potential donor undergoes a CT scan as a part of the screening process. Analytic morphomics has not yet been applied to this population and may be able to help identify potential kidney donors who are at greater risk of developing chronic disease following donation.

The purpose of this study is to examine the potential benefit of analytic morphomics as a long-term risk assessment tool in kidney donors. To this end, we obtained long-term follow-up data from kidney donors at a single large academic institution who donated between 2000 and 2010. Kidney donors were asked to complete a survey that included questions to specifically target the development of cardiovascular risk factors as well as reflective questions about the quality of their donation experience. Analytic morphomic measurements were determined using predonation CT scans. We hypothesize that predonation morphometric measures in kidney donors without preexisting known comorbidities will be associated with the development of chronic disease.

\section{Methods Study Population}

The study population was limited to individuals who underwent donor nephrectomy between January 1, 2000, and September 22, 2010, and had a predonation CT scan on file. Each of the 1038 donors who fit these criteria received a phone call and was asked to complete a brief telephone survey. If patients could not be reached by telephone after multiple attempts, a single letter including the survey was sent to their listed home address. A retrospective chart review was performed to acquire both demographic and laboratory information about each patient. Data collected from the chart review at the time of donation included glomerular filtration rate, creatinine, blood urea nitrogen, $\mathrm{Na}^{+}, \mathrm{K}^{+}, \mathrm{Cl}^{-}, \mathrm{CO}_{2}$, uric acid, blood glucose, $\mathrm{Ca}^{2+}, \mathrm{PO}_{4^{\prime}}$ albumin, protein, alkaline phosphatase, lactate dehydrogenase, aspartate aminotransferase, alanine aminotransferase, total bilirubin, cholesterol, white blood cell count, hemoglobin, hematocrit, and platelet count. Demographic information collected included age, gender, body mass index (BMI), blood type, race, history of smoking, presence of hypertension or cardiovascular disease, and family history of hypertension or cardiovascular disease.

\section{Survey Instrument}

Study subjects who agreed to participate consented according to the standards of the University of Michigan Institutional Review Board. The survey included questions about patients' thoughts on their donation experience and about physical 
health, specifically targeting the onset of various comorbidities (Appendix A). In particular, the survey targeted the onset of cardiovascular risk factors, defined as a patient-reported diagnosis of hypertension, diabetes mellitus, myocardial infarction, kidney disease, or stroke since the time of donation.

\section{Analytic Morphomics}

Aortic calcification, trunk muscle characteristics, and morphometric age are previously validated measures of perioperative outcomes. ${ }^{9-13}$ Aortic calcification was measured on preoperative CT scans using semiautomated methods in MATLAB v13. In brief, the center of the abdominal aorta was first mapped manually between the L1 and L3 vertebrae. Calcification was then defined as having density (Hounsfield units) 25\% higher than the aortic lumen, and a percentage of aortic wall area that reached this threshold was calculated. Percent wall calcification is defined as the calcified area of the aortic wall between L1 and L3 vertebrae divided by the total surface area of the aortic wall between $L 1$ and L3 vertebrae. Semiautomated methods were used to measure trunk muscle characteristics, including psoas muscle cross-sectional area and psoas density at the $L 4$ vertebra. Morphometric age was calculated using a previously validated model that includes total psoas area, psoas muscle density, and the percentage of calcified abdominal aortic wall between $\mathrm{L} 1$ and $\mathrm{L} 3$ vertebrae. This model is designed to redefine an individual's age using the morphometric characteristics of the patient rather than his or her chronological age. A control population of trauma patients was used to design the model. The morphometric age model is described in further detail in our previous work. ${ }^{12}$

\section{Statistical Analysis}

Descriptive statistics were computed for the study cohort. Continuous variables were summarized by mean, standard deviation, and histograms. Comparisons were then made using the Student $t$ test. Categorical variables were compared using the Fisher's exact test.
The effect of abdominal aortic calcification on the development of reported cardiovascular risk factors was determined using the Fischer's exact test. The effect of the extent of aortic wall calcification was determined using logistic regression. Demographic characteristics of our study cohort were compared between calcification and no calcification groups using the Fischer's exact test for dichotomous variables (gender, nonwhite race, smoking history, predonation cardiovascular disease or hypertension, family history of hypertension) and the Student $t$ test for age and BMI. Individuals with nonzero calcification were defined as the calcification group. The odds ratios for chronological age and morphometric age were calculated using univariate logistic regression. To determine whether morphometric age was independently associated with development of cardiovascular risk factors, stepwise logistic regression was performed to identify a set of model covariates from the patient characteristics displayed in Table 1.

All analyses were performed using Stata data analysis and statistical software version 13.1 (StataCorp LP, College Station, Texas). A 2-sided significance of $a=0.05$ was used for all analyses.

\section{Results}

Of the 1038 donors eligible for interview, 613 (59\%) donors who were eligible for interview completed our survey. Of the 613 kidney donors who completed the survey, 598 of these patients (97.6\%) had a CT scan on file that allowed for complete morphometric analysis. These patients composed our study cohort. The average age at the time of donation was $40.7 \pm 11.2$ years, and the average follow-up time was $9.2 \pm 3.0$ years. The average BMI of the study cohort was $27.1 \pm 4.6$, and 367 (61.4\%) donors were female. Table 1 summarizes the demographic characteristics of patients with no calcification versus patients with calcification.

Among the study cohort, 112 (18.7\%) donors had some degree of calcification at the time of their donation. The percent wall calcification 
TABLE 1. Characteristics of Donors With and Without Aortic Calcification at Time of Transplant

\begin{tabular}{|c|c|c|c|c|}
\hline & All Donors & No Calcification & Calcification & $\begin{array}{l}\text { P Value (Calcification } \\
\text { vs No Calcification) }\end{array}$ \\
\hline $\mathrm{n}$ & 598 & $486(81.3 \%)$ & $112(18.7 \%)$ & \\
\hline Age & $40.7 \pm 11.25$ & $38.3 \pm 10.5$ & $51.0 \pm 8.2$ & $<0.001$ \\
\hline Female & $61.4 \%$ & $64.0 \%$ & $50 \%$ & 0.007 \\
\hline Nonwhite race & $96(16.1 \%)$ & $83(17.1 \%)$ & $13(11.6 \%)$ & 0.2 \\
\hline BMI & $27.1 \pm 4.6$ & $27.1 \pm 4.8$ & $27.3 \pm 3.8$ & 0.6 \\
\hline History of smoking & $251(42.0 \%)$ & $177(36.6 \%)$ & $74(66.1 \%)$ & $<0.001$ \\
\hline $\begin{array}{l}\text { Predonation cardio- } \\
\text { vascular disease }\end{array}$ & $25(4.2 \%)$ & $19(3.9 \%)$ & $6(5.4 \%)$ & 0.44 \\
\hline $\begin{array}{l}\text { Predonation } \\
\text { hypertension }\end{array}$ & $19(3.2 \%)$ & $13(2.7 \%)$ & $6(5.4 \%)$ & 0.15 \\
\hline $\begin{array}{l}\text { Family history } \\
\text { of cardiovascu- } \\
\text { lar disease }\end{array}$ & $173(28.9 \%)$ & $123(25.5 \%)$ & $50(44.6 \%)$ & $<0.001$ \\
\hline $\begin{array}{l}\text { Family history of } \\
\text { hypertension }\end{array}$ & $191(31.9 \%)$ & $157(32.6 \%)$ & $34(30.4 \%)$ & 0.74 \\
\hline $\begin{array}{l}\text { Family history of } \\
\text { diabetes mellitus }\end{array}$ & $217(36.3 \%)$ & $173(35.9 \%)$ & $44(39.3 \%)$ & 0.5 \\
\hline
\end{tabular}

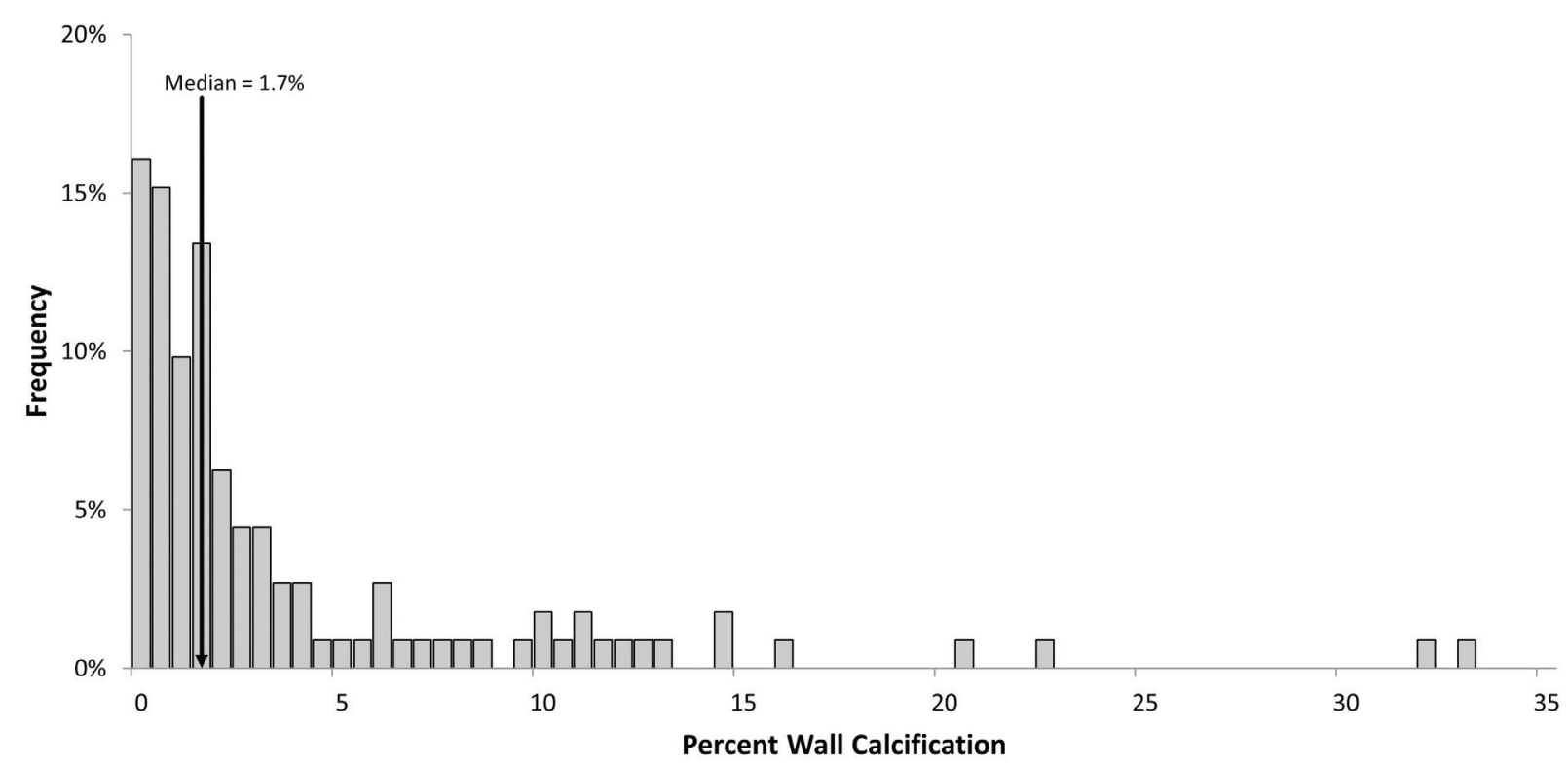

FIGURE 1. Distribution of aortic wall calcification among donors. 
among donors who had calcification ranged from $0.10 \%$ to $33.9 \%$. As shown in Figure 1, the distribution of aortic calcification was skewed to the right with a median of $1.70 \%$ wall calcification.

Compared to individuals with no abdominal aortic calcification, donors with calcification had a significantly higher age at time of transplant (51.0 vs 38.3, $P<0.001)$, an increased history of smoking (66.1\% vs 36.6\%, $P<0.001$ ), and an increased family history of cardiovascular disease (44.6\% vs $25.5 \%, P<0.001)$. In addition, a higher percentage were male (50.0\% vs 36.0\%, $P=0.007)$.

Individuals with any degree of aortic calcification had a higher rate of developing cardiovascular risk factors than those without calcification, but this difference was not statistically significant (20.5\% vs $15.2 \%, P=0.20$ ). As shown in Table 2 , there were no significant differences in development of individual cardiovascular risk factors (diabetes, myocardial infarction, stroke, kidney disease, hypertension) between individuals with and without calcification. Among individuals with calcification, percent wall calcification did not significantly predict the onset of cardiovascular risk factors $(P=0.61)$. Neither total psoas area (males $P=0.068$, females $P=$ 0.482 ) nor psoas density (males $P=0.237$, females $P=0.682$ ) significantly correlated with the development of cardiovascular risk factors.

In univariate analysis, morphometric age was significantly associated with development of cardiovascular risk factors, while chronologic age at time of transplant was not $(P=0.045$ vs $P=$ 0.093). Morphometric age better discriminated patient risk compared to chronologic age $(\mathrm{OR}=$ 1.034 vs $O R=1.017$ ). When comparing the oldest $20 \%$ of morphometric age to the youngest $20 \%$ of morphometric age, individuals in the oldest 20\% developed cardiovascular risk factors with a higher prevalence than the youngest 20\% (22.7 vs 10.8\%, P $=0.016)$. However, this difference was not observed when comparing the oldest $20 \%$ and youngest $20 \%$ of chronologic age (19.5 vs $14.5 \%, P=0.31$ ) (Figure 2). Stepwise logistic regression yielded a model that included history of smoking $(P=$ 0.032, morphometric age $P=0.075)$, and family history of cardiovascular disease $(P=0.273)$. While morphometric age failed to reach nominal significance in this model, it remained in the model upon backward elimination, whereas chronological age did not.

\section{Conclusion}

Analytic morphomics has been shown to be a valuable tool for predicting short- and long-term outcomes in a variety of surgical populations.9-13 We have explored the utility of analytic morphomics in predicting long-term outcomes in living kidney donors. The composite measure of morphomic risk at the time of donation, morphometric age, is associated with the development

\section{TABLE 2. Survey Results}

\begin{tabular}{llll} 
& No Calcification & Calcification & $P$ Value \\
\hline $\mathrm{n}$ & $486(81.3 \%)$ & $112(18.7 \%)$ & \\
Diabetes mellitus & $11(2.3 \%)$ & $4(3.6 \%)$ & 0.50 \\
\hline Hypertension & $63(13 \%)$ & $18(16.1 \%)$ & 0.44 \\
Kidney disease & $5(1.0 \%)$ & $1(0.9 \%)$ & 0.99 \\
Myocardial infarction & $2(0.4 \%)$ & $0(0.0 \%)$ & 0.99 \\
Stroke & $6(1.2 \%)$ & $2(1.8 \%)$ & 0.65 \\
\hline Cardiovascular risk factor & $74(15.2 \%)$ & $23(20.5 \%)$ & 0.20 \\
\hline
\end{tabular}




\section{Age and Cardiovascular Risk Factor Development}

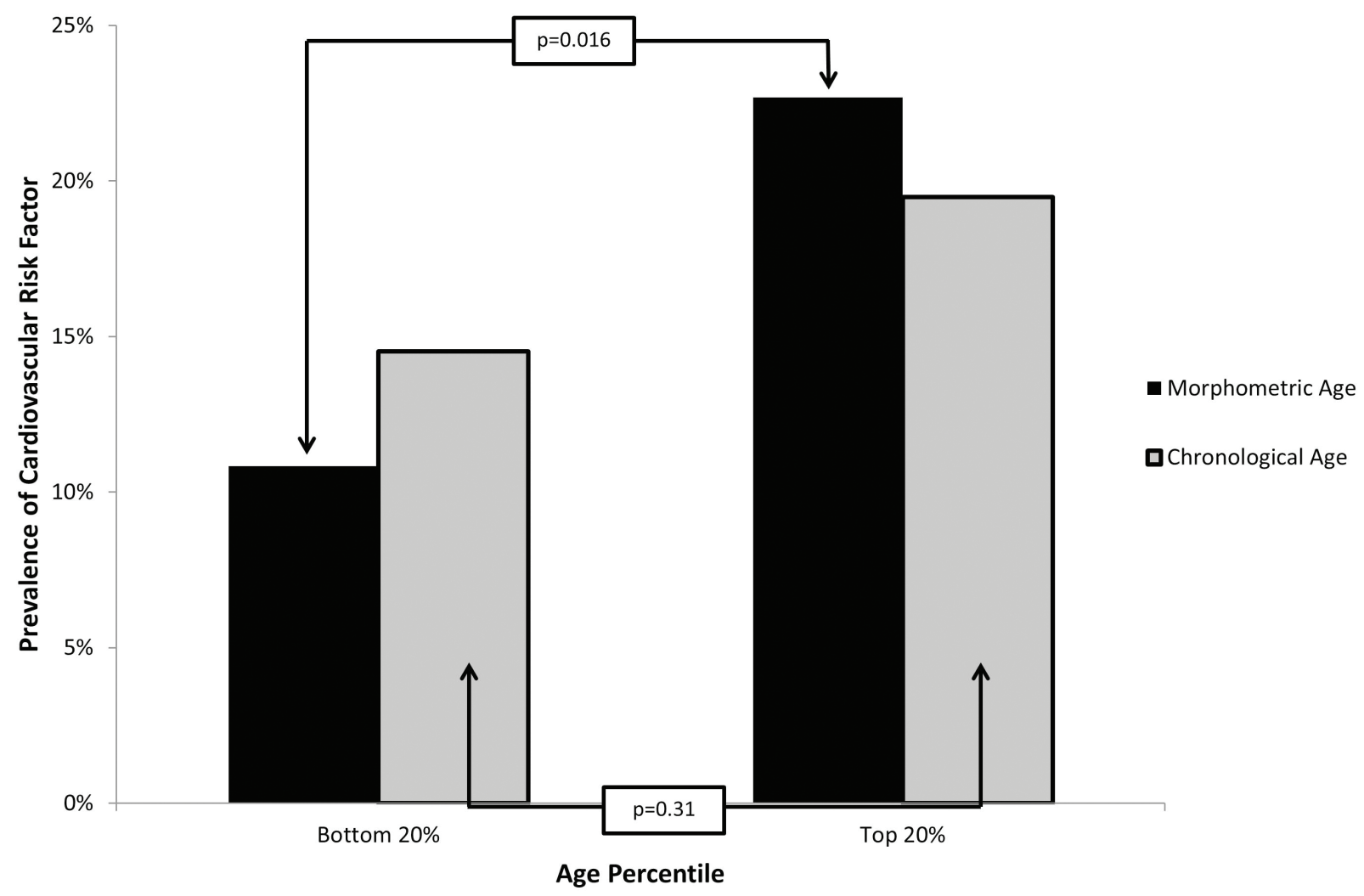

FIGURE 2. Comparison of cardiovascular risk factor prevalence between top and bottom $20 \%$ age groups.

of cardiovascular risk factors following donation. Morphomic measures alone, including vascular wall calcification and trunk muscle size and density, were not significantly associated with the development of cardiovascular risk factors. At this time, it is not clear how morphomic measures will improve the donor selection and informed consent process. With additional work, analytic morphomics may help improve the care of these important patients.

Identifying novel risk factors in the kidney donor population is a top priority. ${ }^{14}$ Over the past decade, older patients and patients with more comorbidities have donated. ${ }^{1}$ There is a growing concern that transplant centers have become too aggressive in their donor assessment policies. Empiric measures of risk are needed for donors. Analytic morphomics may emerge as a useful tool for stratifying risk in kidney donors, many of whom display minimal clinically known risk factors. Previous studies have utilized analytic morphomics to predict adverse outcomes in various surgical populations, including transplant populations. ${ }^{9-13}$ For example, surgical complications in patients with no known cardiovascular risk factors who underwent major elective general or vascular surgery have been shown to correlate with aortic calcification. ${ }^{10}$ In addition, morphometric age was shown to be a predictor of mortality in elective surgical and liver transplant patients.12

Predicting future risk is paramount for kidney donors. The low event rate in the kidney donor population suggests that current donor screening methods are effective. ${ }^{15}$ Conversely, the effects of kidney donation are not fully understood, and long-term follow-up (in excess of 30 years) is necessary. ${ }^{1,16}$ Our work and the work of others note that kidney donors do develop new cardiovascular risk factors following donation. 2-5,15-17 Based on our assessment of a large cohort of donors with an average of 9 years posttransplant, it is not evident 
that novel risk-assessment tools such as analytic morphomics are clinically relevant for risk stratification. More work is needed with larger patient populations, longer-term follow-up, and better analytic morphomic methods.

There are important limitations of this study. This is a retrospective study and is not designed to attribute causality between analytic morphomics and long-term outcomes in kidney donors. Furthermore, this study is also not designed to attribute adverse long-term outcomes to kidney donation as has been explored by other studies. ${ }^{18}$ The results of our study are limited in that our data were collected at a single large academic medical institution that serves as a regional transplant center. Further research with a larger sample size is needed to assess if our findings can be generalized. Additionally, the inherent reporting bias of survey data as well as the selection bias resulting from potential differences between respondents and nonrespondents limits the accurate measurement of cardiovascular risk factors in this population. Incidence of cardiovascular risk factors was patient reported,

\section{References}

1. Living Kidney Donor Follow-Up Conference Writing Group, Leichtman A, Abecassis M, et al. Living kidney donor follow-up: state-of-the-art and future directions, conference summary and recommendations. Am J Transplant. 2011;11(12):25612568.

2. Reese PP, Bloom RD, Feldman HI, et al. Mortality and cardiovascular disease among older live kidney donors. Am J Transplant. 2014;14(8):1853-1861.

3. Fournier C, Pallet N, Cherqaoui Z, et al. Very longterm follow-up of living kidney donors. Transpl Int. 2012;25(4):385-390.

4. Mjoen G, Reisaeter A, Hallan S, et al. Overall and cardiovascular mortality in Norwegian kidney donors compared to the background population. Nephrol Dial Transplant. 2012;27(1):443-447.

5. Garg AX, Meirambayeva A, Huang A, et al. Cardiovascular disease in kidney donors: matched cohort study. BMJ. 2012;344:e1203.

6. Tong A, Chapman JR, Wong G, de Bruijn J, Craig JC. Screening and follow-up of living kidney donors: and individuals did not return to the hospital for a comprehensive clinical assessment. Follow-up clinical appointments would have provided more accurate reporting but were beyond the scope of this study. Despite a high survey response rate (57.6\%), there was a low event rate over the mean 9 years of follow-up in this population. Kidney donors are typically followed less closely than recipients for numerous reasons. However, relaxation of kidney donation criteria over the last decade may warrant greater follow-up. This sustained evaluation would allow time-to-event statistical analysis. Finally, donation criteria changed within our center over the course of the study period, calling into question whether observations based on these data are relevant for today's practice. These limitations stress the need for more stringent communication with living kidney donors.

Transplant clinicians should continue to try to improve predonation risk assessment. Analytic morphomics does not clearly add to current methods of risk stratification in kidney donors, but it does show promise as a novel risk assessment tool.

a systematic review of clinical practice guidelines. Transplantation. 2011;92(9):962-972.

7. Fishbane S. Cardiovascular risk evaluation before kidney transplantation. J Am Soc Nephrol. 2005;16(4): 843-845.

8. Garg AX, Prasad GV, Thiessen-Philbrook HR, et al. Cardiovascular disease and hypertension risk in living kidney donors: an analysis of health administrative data in Ontario, Canada. Transplantation. 2008;86(3):399-406.

9. Englesbe MJ, Lee JS, He K, et al. Analytic morphomics, core muscle size, and surgical outcomes. Ann Surg. 2012;256(2):255-261.

10. Harbaugh CM, Terjimanian MN, Lee JS, et al. Abdominal aortic calcification and surgical outcomes in patients with no known cardiovascular risk factors. Ann Surg. 2013;257(4):774-781.

11. Lee JS, He K, Harbaugh CM, et al. Frailty, core muscle size, and mortality in patients undergoing open abdominal aortic aneurysm repair. J Vasc Surg. 2011;53(4):912-917. 
12. Waits SA, Kim EK, Terjimanian MN, et al. Morphometric age and mortality after liver transplant. JAMA surg. 2014;149(4):335-340.

13. Canvasser LD, Mazurek AA, Cron DC, et al. Paraspinous muscle as a predictor of surgical outcome. J Surg Res. 2014;192(1):76-81.

14. Segev DL, Muzaale AD, Caffo BS, et al. Perioperative mortality and long-term survival following live kidney donation. JAMA. 2010;303(10):959-966.

15. Morgan BR, Ibrahim HN. Long-term outcomes of kidney donors. Curr Opin Nephrol Hypertens. 2011;20(6):605-609.
16. Gossmann J, Wilhelm A, Kachel HG, et al. Long-term consequences of live kidney donation follow-up in 93\% of living kidney donors in a single transplant center. Am J Transplant. 2005;5(10):2417-2424.

17. Garg AX, Nevis IF, McArthur E, et al. Gestational hypertension and preeclampsia in living kidney donors. N Eng/ J Med. 2015;372(2):124-133.

18. Ibrahim HN, Foley R, Tan L, et al. Long-term consequences of kidney donation. N Engl J Med. 2009;360(5):459-469. 